\title{
Reutilización de un residuo de la industria petrolera (FCC) en la producción de elementos constructivos ${ }^{1}$ \\ Reusing a residue of the oil industry (FCC) in the production of building elements ${ }^{2}$
}

\author{
Eduard A. Caicedo-Caicedo ${ }^{3}$ \\ Ruby Mejía-de-Gutiérrez ${ }^{4}$ \\ Marisol Gordillo-Suárez ${ }^{5}$ \\ Janneth Torres-Agredo ${ }^{6}$
}

doi: 10.11144/Javeriana.iyu19-1.rrip

\section{Cómo citar este artículo:}

E. Caicedo, R. Mejía, M. Gordillo y J. Torres, "Reutilización de un residuo de la industria petrolera (FCC) en la producción de elementos constructivos", Ing. Univ., vol. 19, no. 1, pp. 135-154, Ene.-Jun., 2015.

\footnotetext{
' Fecha de recepción: 7 de febrero de 2013. Fecha de aceptación: 24 de noviembre de 2014. Este artículo se deriva de un proyecto de investigación titulado "Estudio del comportamiento de morteros y concretos adicionados con catalizador de craqueo catalítico usado (FCC)", registro 101-452-21170, desarrollado por los grupos de investigación Materiales Compuestos, de la Universidad del Valle (CENM), Cali, y Materiales y Medio Ambiente, de la Universidad Nacional, Sede Palmira, Colombia, financiado por Colciencias.

${ }^{2}$ Submitted on: February $7^{\text {th }}, 2013$. Accepted on: November $24^{\text {th }}, 2014$. This article is the result of the research project Study of the behavior of mortars and concrete containing spent cracking catalyst (FCC), registration number 101-452-21170, developed by the research groups: Composite Materials of the Universidad del Valle (CENM), Cali, and Materials and the Environment of the Universidad Nacional de Colombia, financed by Colciencias.

${ }^{3}$ Ingeniero de materiales, magíster en Ingeniería, Universidad del Valle, Cali, Colombia. Correo electrónico: casso39@hotmail.com. ${ }^{4}$ Doctora en Ciencias Químicas, Universidad Complutense, Madrid, España. Profesora titular, Escuela de Ingeniería de Materiales, Universidad del Valle. Correo electrónico: ruby.mejia@correounivalle.edu.co.

${ }^{5}$ Doctora en Ingeniería con énfasis en Ingeniería de Materiales, Universidad del Valle, Cali, Colombia. Profesora de la Facultad de Ciencias, Universidad Autónoma de Occidente, Cali, Colombia. Correo electrónico: mgordillo@uao.edu.co.

${ }^{6}$ Doctora en Ingeniería con énfasis en Ingeniería de Materiales, Universidad del Valle, Cali, Colombia. Profesora a sociada, Universidad Nacional de Colombia, sede Palmira, Colombia. Correo electrónico: jtorres@unal.edu.co
} 


\section{Resumen}

Este trabajo analiza la viabilidad de utilizar un residuo de catalizador gastado (FCC) del proceso de craqueo catalítico, procedente de una empresa petrolera colombiana, en la producción de elementos constructivos, tipo bloques y adoquines. Para definir la mezcla óptima de cemento/FCC, se preparan morteros de cemento Portland con proporciones de FCC entre 0 y $70 \%$, como reemplazo del cemento, y se evalúa su resistencia a compresión a edades hasta de 28 días de curado. Mediante un procesamiento estadístico, aplicando la metodología de respuesta, se optimizan las proporciones de cada componente en la mezcla, para su posterior implementación en la producción de bloques y adoquines vibrocompactados. Complementariamente, se realiza el estudio del proceso de hidratación mediante técnicas de difracción de rayos $\mathrm{X}(\mathrm{DRX})$ y análisis térmico diferencial (TG/DTG). Los resultados indican que es posible incorporar FCC al concreto, sustituyendo el cemento hasta en $45 \%$, para obtener un elemento con características mecánicas comparables a los disponibles comercialmente. En la norma colombiana actual, los bloques desarrollados se clasifican como bloques no estructurales, y los adoquines, como adoquines de piso. Se plantea este proceso como una alternativa de utilización del subproducto y un aporte a la sostenibilidad ambiental.

\section{Palabras clave}

Catalizador gastado de craqueo catalítico; cementos adicionados; elementos constructivos

\section{Abstract}

This paper analyzes the feasibility of using a residue of spent catalyst (FCC) of the cracking process, from a Colombian oil company, in the production of building elements such as blocks and pavers. To define the optimal mix of Portland cement / FCC, Portland cement mortars with FCC ratios between 0 and $70 \%$ as replacement of cement were prepared and its compressive strength is evaluated at ages up to 28 days of curing. Using a statistical processing, applying the methodology of response, the proportions of each component in the mixture are optimized. Subsequently, blocks and pavers were developed using a vibro-compaction machine. In addition the study of the process of hydration is performed by techniques of $\mathrm{X}$-ray diffraction (XRD) and differential thermal analysis (TG / DTG). The results indicate that it is possible to incorporate into the concrete FCC as replacement of cement up to $45 \%$ and obtain a building element with mechanical properties comparable to those commercially available elements. According to the Colombian standard, the elements developed in this research classified as non-structural blocks and floor pavers. It raises this is an alternative use of the residue and contribute to environmental sustainability.

\section{Keywords}

Fluid catalytic cracking; blended cements; building elements 


\section{Introducción}

Uno de los procesos industriales que más producen gases de efecto invernadero (GEI) es la producción de cemento; en términos generales, por cada tonelada de cemento Portland (OPC) se generan aproximadamente 0,87 toneladas de $\mathrm{CO}_{2}$ [1]. Este valor es atribuible a la decarbonatación de la materia prima caliza en el proceso de producción de clinker y al uso de combustibles fósiles en los hornos de clinkerización. Adicionalmente, en el proceso de producción de cemento se registra un elevado consumo de recursos naturales y de energía; este último relacionado con las altas temperaturas requeridas en los procesos de clinkerización (1400-1500 ${ }^{\circ} \mathrm{C}$ ) y con el gasto energético asociado a los procesos de molienda. Debido a lo anterior, y tomando en consideración la producción mundial de cemento, que para 2013 fue de aproximadamente 4.000 Mton [2], la investigación acerca de materiales alternativos para incorporar o sustituir al cemento se ha constituido en una línea de investigación de gran interés científico y tecnológico a escala mundial y ha sido el principal objetivo de muchos grupos de investigación. Entre estos materiales alternativos cabe mencionar las puzolanas naturales, las escorias siderúrgicas de alto horno, el humo de sílice, las cenizas volantes, el metacaolín y la ceniza de cascarilla de arroz, entre otros [3]-[17]. Algunos de estos materiales han sido incluidos en las especificaciones y normativas de algunos países y han dado lugar a los denominados cementos adicionados o compuestos (blended cements).

Precisamente, el catalizador usado del proceso de craqueo catalítico (FCC, del inglés fluid catalytic cracking) es un residuo industrial de las refinerías de petróleo en las unidades de ruptura catalítica en lecho fluido; está compuesto, principalmente, por sílice y alúmina, posee partículas esféricas de alta reactividad con poros de gran tamaño y una estructura cristalina zeolítica, tipo faujasita [18]. Este catalizador es usado inicialmente en la unidad de craqueo, con el fin de romper las moléculas de hidrocarburo para convertirlas en unas más pequeñas y de mayor valor agregado, como las gasolinas; una vez este material pierde su actividad se convierte en desecho. 
A escala mundial, tomando en consideración la capacidad de procesamiento de crudo y la de procesamiento en craqueo catalítico, se estima un consumo de catalizador de aproximadamente 400.000 ton/año [19]. En Colombia la empresa Ecopetrol, encargada del procesamiento de los combustibles, dispone de dos complejos industriales de refinación, ubicados en Barrancabermeja y Cartagena, donde cuenta en total con cinco unidades de craqueo catalítico en lecho fluido. Al tomar en cuenta el consumo diario ( 3 a 7 ton/día por barril), las pérdidas y la reposición de catalizador en el proceso, se estima que la generación del FCC es, aproximadamente, 15.000 toneladas al año [20]. Actualmente Ecopetrol entrega parte del catalizador usado a pequeñas refinerías que aprovechan su escasa actividad ya degradada, o a industrias que lo emplean como relleno en sus procesos o como elemento inerte para pavimentación y rellenos de caminos, y en algunos casos la empresa que lo genera debe disponerlo. Esto significa que sus propiedades potenciales, una vez es desechado como catalizador de refinación, no están siendo aprovechadas, aunque existe la posibilidad de incorporarlo como materia prima en diferentes campos. Este material ha sido caracterizado como residuo no peligroso [21], [22] y puede ser usado potencialmente en la manufactura de materiales resistentes al fuego o adicionado a las arcillas para producir tejas cerámicas, ladrillos refractarios y ladrillos aislantes, o incorporado como relleno en productos asfálticos, entre otros usos [20], [23], [24]. Adicionalmente, es considerado un material puzolánico muy activo, capaz de combinar el hidróxido de calcio liberado en la hidratación del cemento Portland y formar compuestos de carácter hidráulico que aportan propiedades mecánicas y de durabilidad al concreto. En general, se recomienda utilizar un porcentaje de adición de FCC de hasta $15 \%$ [19], [25]-[37].

El presente artículo tiene como objetivo, a partir de estudios previos conducentes al desarrollo de morteros y concretos de alto desempeño, evaluar el uso en altos porcentajes de este subproducto industrial como reemplazo parcial del cemento en volúmenes elevados para la producción de elementos constructivos tipo bloques y adoquines.

\section{Materiales y métodos experimentales}

\subsection{Selección y caracterización de materiales}

En este estudio se utilizó un FCC del proceso de craqueo catalítico, procedente de una empresa petrolera colombiana y un cemento Portland tipo 1 (OPC). En la tabla 1 se incluyen las características químicas de dos muestras del material, identificadas como FCC-1 y FCC-2. Se aprecia que el FCC está compuesto mayo- 
ritariamente por alúmina y sílice, en una cantidad cercana al $90 \%$. Cabe anotar que la composición es similar a la informada por otros autores y se asemeja a la del metacaolín (MK), material puzolánico de alta actividad; en consecuencia, es de esperarse que se presenten comportamientos similares a los obtenidos en mezclas de cemento Portland y MK. [6], [28], [36], [38]. La distribución y tamaño de las partículas fue determinada por granulometría láser en un equipo Mastersizer 2000 (Malvern Instrument). Los resultados obtenidos se presentan en la figura 1, en la cual se puede observar un tamaño medio de partícula de 15,50 $\mu \mathrm{m}, 16,15 \mu \mathrm{m}$ y $52,60 \mu \mathrm{m}$, para OPC, FCC-1 y FCC-2, respectivamente. El cemento seleccionado para este estudio corresponde al disponible comercialmente para obras de hormigón en general, al que no se le exigen propiedades especiales.

Tabla 1. Composición química de los materiales utilizados

\begin{tabular}{|l|c|c|c|}
\hline \multicolumn{1}{|c|}{ Componente } & OPC & FCC-1 & FCC - 2 \\
\hline $\mathrm{SiO}_{2}(\%)$ & 21,17 & 44,13 & 48,09 \\
\hline $\mathrm{Al}_{2} \mathrm{O}_{3}(\%)$ & 6,02 & 46,06 & 41,57 \\
\hline $\mathrm{Fe}_{2} \mathrm{O}_{3}(\%)$ & 4,06 & 0,81 & 0,91 \\
\hline $\mathrm{CaO}(\%)$ & 55,44 & 0,52 & 0,22 \\
\hline $\mathrm{MgO}(\%)$ & 1,33 & - & 0,13 \\
\hline $\mathrm{K}_{2} \mathrm{O}(\%)$ & - & 0,11 & 0,09 \\
\hline $\mathrm{TiO}_{2}(\%)$ & - & 0,67 & 0,85 \\
\hline Pérdidas por ignición (\%) & 7,84 & 6,50 & 2,19 \\
\hline
\end{tabular}

OPC: Cemento Portland Ordinario; FCC-1 y FCC-2: Muestras del catalizador gastado. Fuente: presentación propia de los autores

La figura 2 presenta el análisis de difracción de rayos X (DRX) del FCC, realizado en un equipo RX Rigaku RINT 2200. En este análisis se puede apreciar la naturaleza parcialmente cristalina del material; se identifican picos de un material aluminosilicato sódico hidratado de naturaleza zeolítica y de carácter similar al de la faujasita $(\mathrm{F})\left(2 \mathrm{q}=6,19^{\circ} ; 15,6^{\circ} ; 2,58^{\circ}\right)$, además de cuarzo $(\mathrm{Q})$ y caolinita (K) [22], [25], [39], [40].

Se resalta una leve diferencia de amorficidad entre FCC1 y FCC2, representada por un levantamiento suave de la línea base del difractograma para los ángulos $2 \theta$ ubicados en la región entre $20^{\circ}$ y $30^{\circ}$. Aunque en este estudio no se evaluó la actividad puzolánica del material FCC, estudios anteriores en FCC proveniente de la misma fuente, aplicando la norma ASTM C311, han informado acerca de valores de hasta $105 \%$, lo cual supera ampliamente lo especificado por la norma 
(75\%) para catalogar un material como puzolánico [41], [42]. Asimismo, mediante la técnica de TCLP (del inglés toxicity characteristic leaching procedure), aplicada a morteros adicionados con $20 \%$ de FCC respecto a la cantidad de cemento, se ha demostrado que el catalizador usado no representa un problema desde el punto de vista de la lixiviación de elementos, como As, $\mathrm{Pb}, \mathrm{Zn}, \mathrm{Cr}$ y La, lo cual hace que este subproducto pueda ser considerado residuo no peligroso, lo cual confirma estudios realizados con FCC de diversa procedencia [21], [22], [36], [42].

Figura 1. Distribución de tamaño de partícula de los materiales utilizados

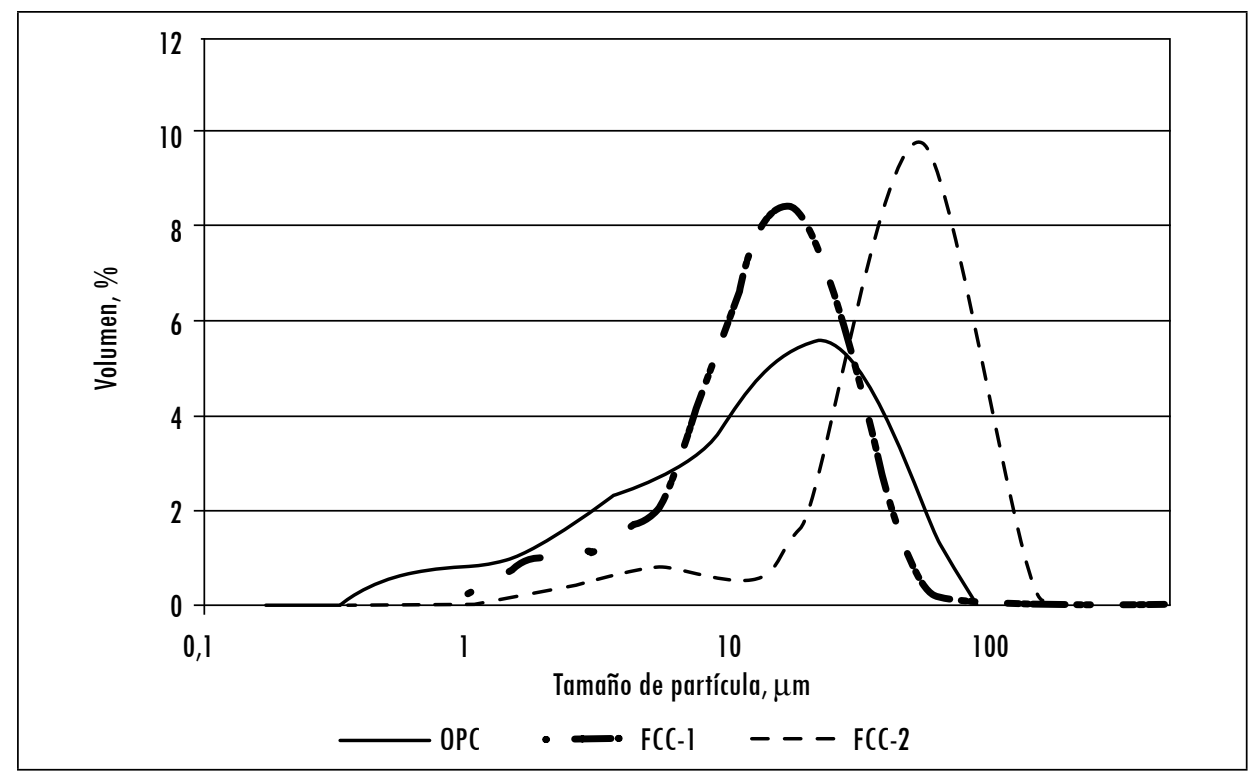

Fuente: presentación propia de los autores

\subsection{Fabricación de especímenes y optimización de mezclas}

Para la optimización estadística, se produjeron especímenes cilíndricos de mortero de $3 \mathrm{~cm}$ de diámetro y $6 \mathrm{~cm}$ de altura, con relación cementante:árido 1:2,75. La relación a/C se ajustó para obtener una fluidez entre 105 y 110 [43]. Cabe anotar, que en esta relación, $C$ representa la suma de las cantidades de OPC y FCC-1 presentes en la mezcla. Como árido fino en los morteros se utilizó una arena proveniente de canto rodado con un módulo de finura de 2,05. Los especímenes fueron sometidos a curado y, posteriormente, ensayados en compresión a edades de 7 y 28 días, en una prensa universal Instron serie 3369 de $50 \mathrm{kN}$ de carga máxima, a una velocidad de carga de $1 \mathrm{~mm} / \mathrm{min}$. Los resultados de resistencia corresponden al promedio de tres especímenes ensayados por mezcla y por edad de curado. 
Figura 2. Espectro DRX para FCC-I y FCC-2

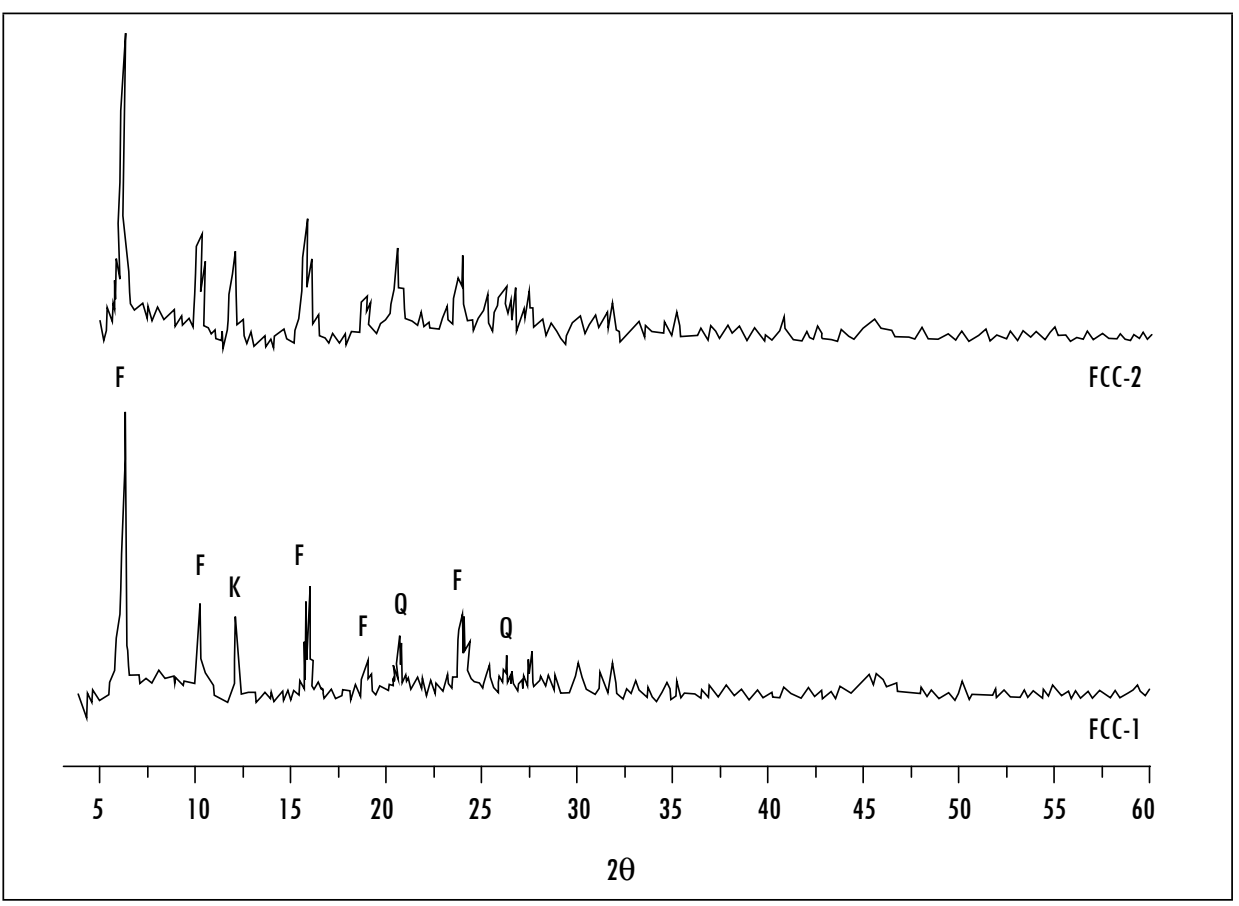

Fuente: presentación propia de los autores

Para el análisis estadístico, en un primer estudio se sustituyó parcialmente el OPC por FCC de manera aleatoria, en proporciones de 10, 40 y $70 \%$, con el fin de optimizar la mezcla cementante. Después se utilizó un diseño de mezclas de vértices extremos, con proporciones entre 30 y $60 \%$ de FCC como reemplazo del cemento. En ambos casos se consideraron dos variables independientes X1: OPC y X2: FCC, y como variable de respuesta $(\mathrm{Y})$, la resistencia máxima a compresión de las muestras a 28 días de curado. Los componentes del diseño, el rango de las proporciones y las respectivas codificaciones para cada mezcla se presentan en la tabla 2.

Tabla 2. Componentes y proporciones del diseño estadístico

\begin{tabular}{|l|c|c|c|c|c|c|c|c|c|c|c|}
\hline \multicolumn{2}{|c|}{ Componentes } & \multicolumn{6}{|c|}{ Mezclas preliminares } & \multicolumn{7}{|c|}{ Proporciones de acotación } \\
\hline X1 & OPC & 0,90 & 0,60 & 0,30 & 0,70 & 0,62 & 0,60 & 0,55 & 0,50 & 0,47 & 0,40 \\
\hline X2 & FCC & 0,10 & 0,40 & 0,70 & 0,30 & 0,38 & 0,40 & 0,45 & 0,50 & 0,53 & 0,60 \\
\hline \multicolumn{2}{|l|}{ Codificación } & P10 & P40 & P70 & M30 & M38 & M40 & M45 & M50 & M53 & M60 \\
\hline
\end{tabular}

OPC: Cemento Portland Ordinario; FCC: Catalizador gastado.

Fuente: presentación propia de los autores 
La variable de respuesta puede ser expresada en función de las variables independientes $\left(X_{i}\right)$, esto es, $Y=\mathrm{F}\left(X_{1}, X_{2}\right)$. La superficie generada por la función anterior puede ser descrita en tres dimensiones y está determinada por la ecuación 1.

$$
Y=\beta_{0}+\beta_{1} X_{1}+\beta_{2} X_{2}+\beta_{3} X_{1}^{2}+\beta_{4} X_{2}^{2}+\beta_{5} X_{1} X_{2}+\varepsilon
$$

Donde, $Y$ es la variable de respuesta, resistencia a la compresión (MPa); $\beta_{i}$ son los coeficientes de la regresión; $X_{1}$ es la proporción de OPC; $X_{2}$ es la correspondiente a FCC; $X_{1}^{2} \mathrm{y}_{2}{ }^{2}$ son los efectos cuadráticos; $X_{1} X_{2}$ es el efecto de interacción entre OPC y FCC; y $\varepsilon$ es el error experimental.

\subsection{Fabricación de elementos constructivos y ensayos}

Con los resultados de la optimización se elaboraron elementos constructivos de carácter demostrativo. Para ello se diseñó un concreto de $380 \mathrm{~kg} / \mathrm{m}^{3}$ de cementante a partir de la mezcla de OPC y FCC-2, utilizando una relación a/C de 0,4. Esta relación se seleccionó con el fin de obtener una mezcla seca. Como agregado se utilizó una mezcla de áridos finos y gruesos de canto rodado, con una relación agregado/cementante en la mezcla de 4,86. Se realizó la mezcla manual de estos materiales y se conformaron los elementos por vibrocompactación [44]. Los elementos constructivos son bloques huecos (B) y adoquines $(A)$. Los bloques huecos tienen un área transversal de $0,4 \times 0,10$ $\mathrm{m}$, una altura de $0,2 \mathrm{~m}$, un ancho de tabique de 0,02 $\mathrm{m}$ y están definidos por la norma NTC 4076; los adoquines poseen un rectángulo inscrito de 0,2 ×0,09 m, una altura de 0,08 m y están definidos en la norma NTC 2017. Para efectos de comparación, se utilizaron bloques comerciales identificados como BC, con un área transversal de 0,4 $\times 0,12 \mathrm{~m}$, una altura de 0,2 $\mathrm{m}$ y un ancho de tabique de $0,025 \mathrm{~m}$. La figura 3 muestra los elementos desarrollados (3a y 3b) y los de referencia (3c).

Los elementos son curados bajo agua durante 28 días, para luego evaluar sus propiedades: resistencia a la compresión en bloques y cilindros, resistencia a flexotracción en adoquines y absorción. El montaje de los ensayos mecánicos se presenta en la figura 4.

Los resultados obtenidos se compararon con los presentados por los elementos de referencia. Para cada elemento se ensayaron tres especímenes; el resultado reportado corresponde al promedio de los valores obtenidos en el ensayo. 
Figura 3. (a) Bloques producidos, (b) adoquines tipo I y (c) bloques de referencia

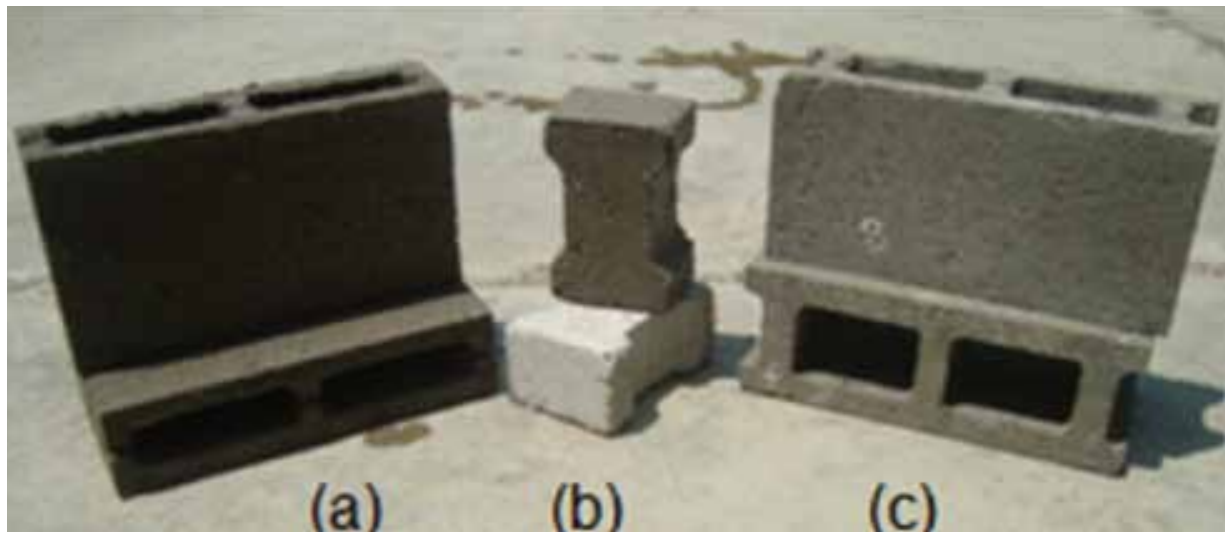

Fuente: presentación propia de los autores

Figura 4. Montaje de los ensayos a compresión y flexión

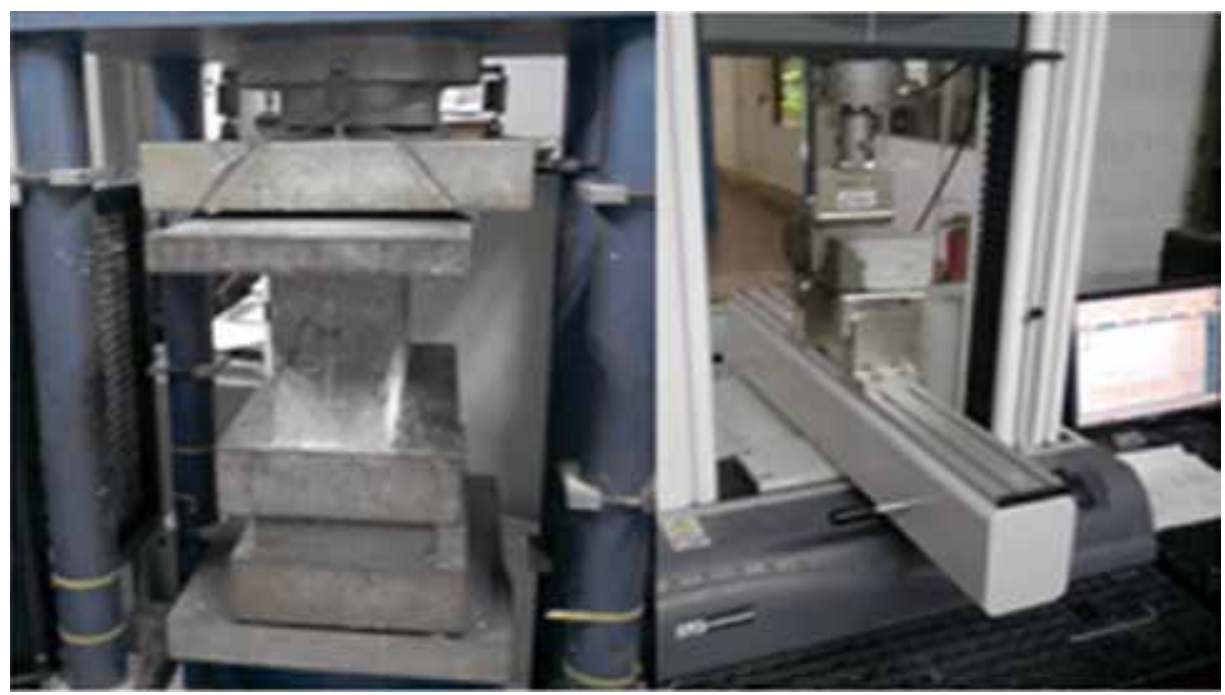

Fuente: presentación propia de los autores

\subsection{Ensayos complementarios}

Igualmente, se determinaron los productos de hidratación de OPC+FCC-1. Para ello se prepararon pastas de cemento Portland y FCC-1, en proporción de 0,33 y $50 \%$, con una relación a/C de 0,4. Estas pastas, luego de curadas a 28 días bajo agua, se sometieron a un proceso de molienda y, posteriormente, a un tratamiento químico con acetona y etanol, con el fin de parar la reacción 
de hidratación. El seguimiento y la evaluación del proceso de hidratación se realizaron aplicando las técnicas de DRX y de termogravimetría (TG) y su correspondiente derivada (DTG); en este último caso se utilizó un termogravímetro TA Instruments SDT Q600.

\section{Resultados y discusión}

\subsection{Resistencia mecánica a compresión: Análisis estadístico en morteros y optimización de las mezclas}

La tabla 5 presenta los resultados de la resistencia a compresión durante 28 días de curado de los especímenes de morteros OPC+FCC-1, identificados en la tabla 2 como P10, P40 y P70; estos resultados muestran que existe un valor óptimo de adición para obtener una resistencia similar o superior a la reportada por el cemento de referencia a la misma edad de curado (17,320 MPa). Estadísticamente se utilizó la función de utilidad, con parámetros de optimización de mínima resistencia 4,2 $\mathrm{MPa}$, y con un valor objetivo de $20 \mathrm{MPa}$, con lo cual se obtuvieron como proporciones óptimas OPC/ FCC de 67 y $33 \%$ respectivamente, con una resistencia de 19,246 MPa y una predicibilidad de 0,95 , como se aprecia en la misma figura 5. Cabe anotar que experimentalmente para esta mezcla se obtuvo un valor de resistencia de 18,8 MPa.

Figura 5. Optimización de la mezcla, resistencia a 28 días de curado

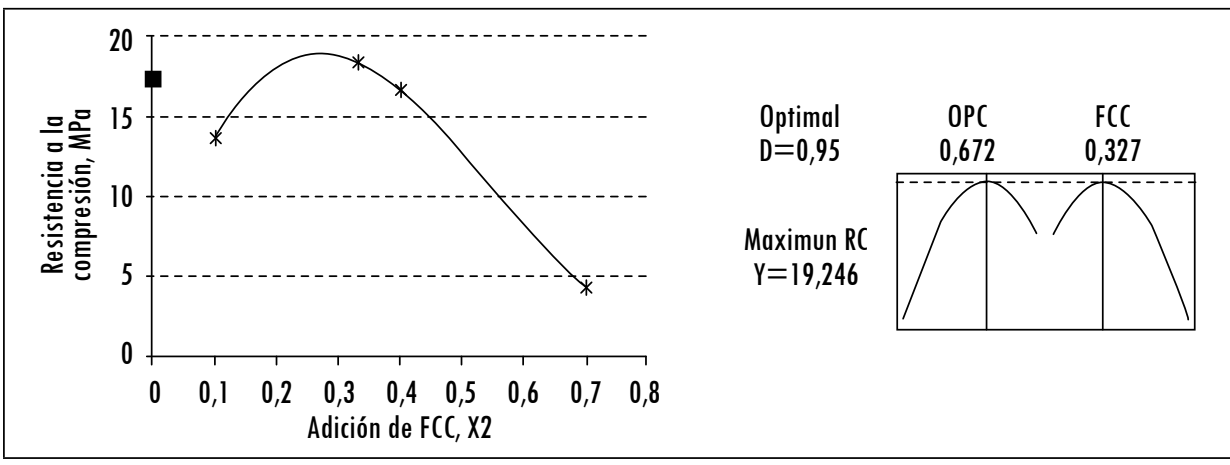

Fuente: presentación propia de los autores

En general, las mezclas evaluadas (rango 30-60 \% FCC) muestran un desarrollo resistente acorde con el porcentaje de adición, como se observa en la figura 6 , en la cual se representan los promedios de los ensayos realizados sobre tres especímenes de cada mezcla, a edades de curado de 7, 14 y 28 días; 
cabe anotar que la desviación estándar de los datos experimentales fluctúa entre 0,14 y 3,67 .

En general, se aprecia que en los cementos con adición de FCC el desarrollo de la resistencia a la edad de 7 días de curado, comparado a la reportada a 28 días fue de hasta $70 \%$, lo cual es indicativo de la buena calidad como material puzolánico de la adición utilizada [41].

Figura 6. Resultados experimentales de resistencia a compresión en función de la edad de curado y del porcentaje de adición de FCC

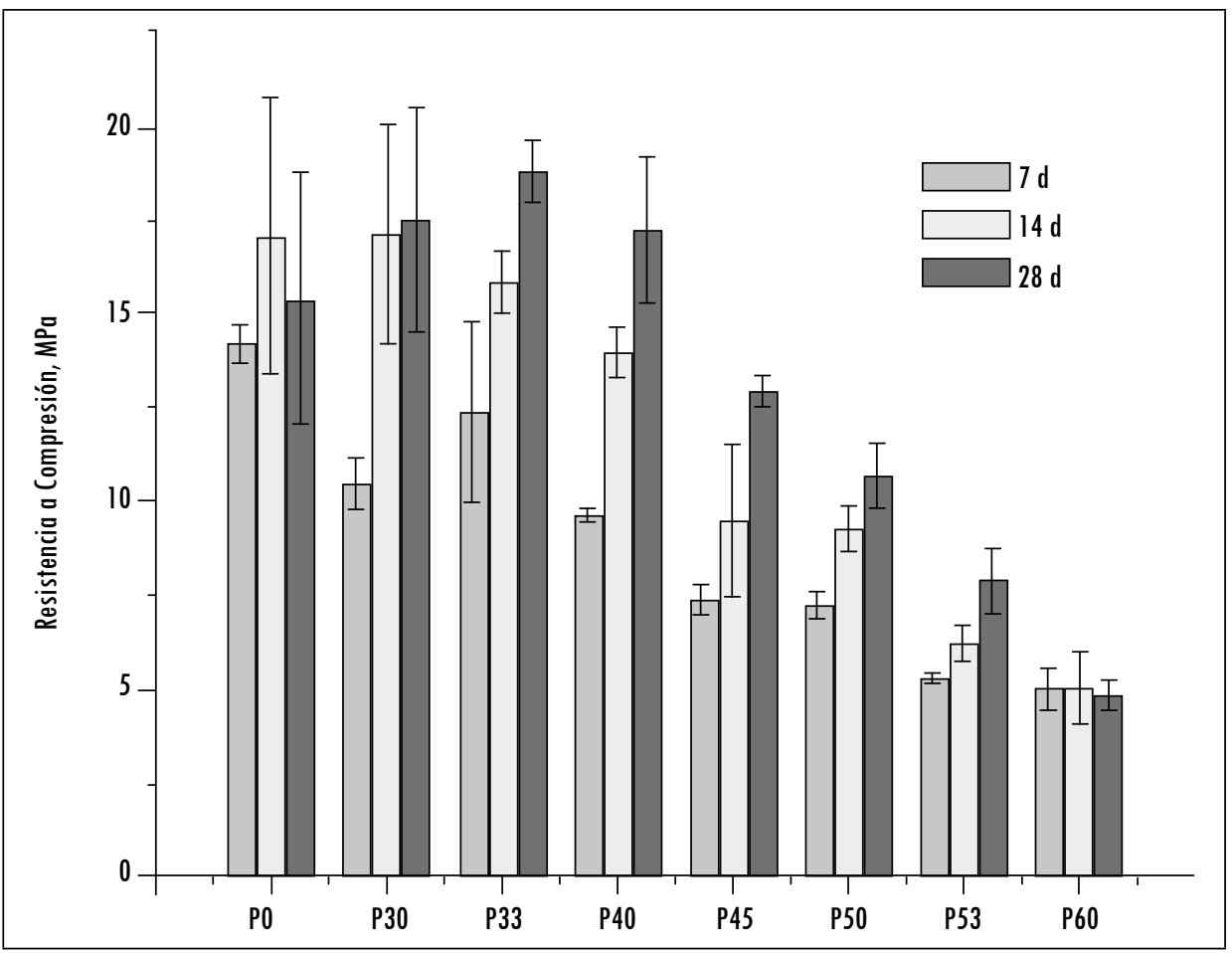

Fuente: presentación propia de los autores

En la figura 7 se expresa un índice resistente, calculado como la relación porcentual entre la resistencia del material adicionado (OPC+FCC) y el material sin adición (OPC) luego del procesamiento estadístico de los resultados experimentales. Aquí se aprecia que es posible obtener una resistencia superior a la mezcla de referencia utilizando porcentajes de adición en el rango de 18,5 a $34 \%$ FCC; al incrementar el porcentaje de adición hasta $45 \%$, se obtienen valores superiores al $75 \%$ de la resistencia del mortero sin adición (OPC), 
tomando en consideración la norma ASTM C628 para materiales puzolánicos. Estos valores de sustitución aquí reportados difieren de los resultados obtenidos en la mayoría de las investigaciones realizadas anteriormente, en las cuales, para cementos Portland sustituidos por FCC se recomiendan valores entre 10 y $25 \%$ [18], [22], [26], [30], [36], [45], [46].

Sin embargo, cabe anotar que la mayoría de los estudios se han enfocado en obtener máxima resistencia y han tomado como punto de partida investigaciones previas con adiciones puzolánicas que, en general, han limitado los porcentajes de adición a órdenes menores al $20 \%$, tal es el caso del metacaolín o el humo de sílice.

En la presente investigación, debido a que el objetivo final es la aplicación del FCC en la producción de bloques y adoquines y a que este tipo de elementos no requiere órdenes de resistencia elevada, es posible el uso de mayores proporciones de adición en la mezcla. Es importante aclarar que el porcentaje óptimo en cada caso dependerá del tipo de cemento utilizado, y en particular, de la disponibilidad de portlandita para la reacción puzolánica.

Figura 7. Incremento resistente de mezclas de $\mathrm{OPC} / \mathrm{FCC}-1$, a 28 días de curado

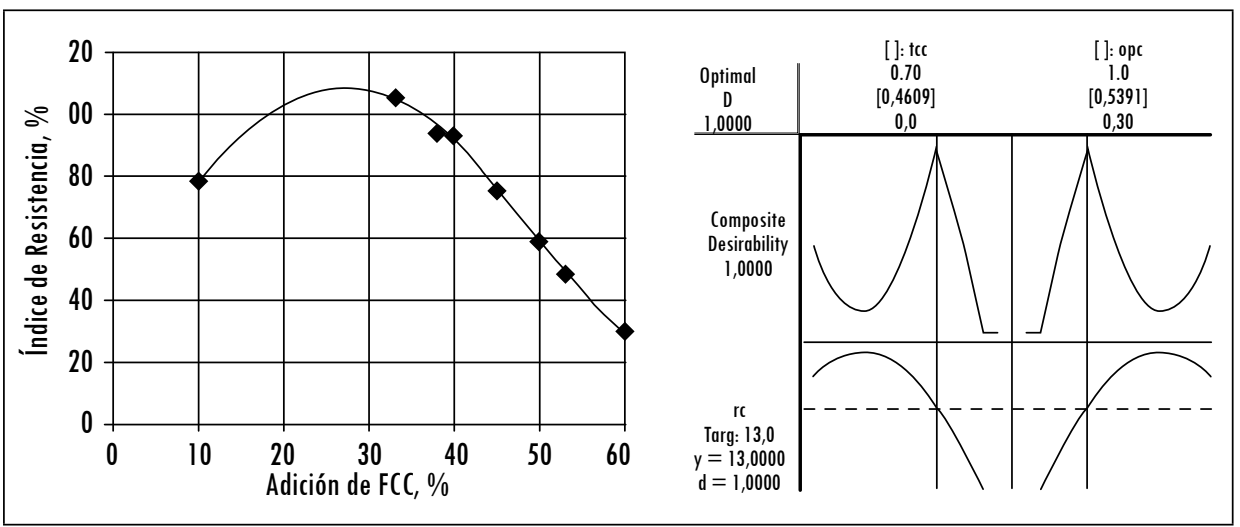

Fuente: presentación propia de los autores

\subsection{Fabricación de adoquines y bloques}

Con fundamento en los análisis anteriores, para la fabricación de adoquines (A) y los bloques (B) de concreto se evaluaron —además de la proporción definida como óptima (67\% OPC/33\% FCC) y de la mezcla de referencia (OPC 0\% de adición), identificadas como B33 y B0, respectivamente - tres mezclas con proporciones de $39 \%$ (B39), $45 \%$ (B45) y $50 \%$ (A50). 
El desempeño resistente de estas mezclas difiere de las evaluadas en morteros, lo cual, entre otros factores de diseño y composición, es atribuible al cambio de material FCC en esta etapa de la experimentación, pues se utilizó FCC-2 (tabla 1), cuyo tamaño de partícula es superior a FCC-1. La tabla 3 muestra los resultados de resistencia mecánica a compresión ( $\mathrm{R}_{\mathrm{C}}$, bloques) y flexión ( $\mathrm{R}_{\mathrm{F}}$, adoquines), absorción y densidad aparente, correspondientes a los elementos constructivos fabricados. En la misma tabla se incluyen, con propósitos de comparación, resultados obtenidos en muestras comerciales de bloques (BC1 y BC2).

Tabla 3. Propiedades mecánicas y físicas de los bloques

\begin{tabular}{|c|c|c|c|}
\hline Código & $\begin{array}{l}R_{c} 28 d \\
(\mathrm{MPa})\end{array}$ & $\begin{array}{c}\text { Absorción } \\
(\%)\end{array}$ & $\begin{array}{c}\text { Densidad aparente } \\
\left(\mathrm{kg} / \mathrm{m}^{3}\right)\end{array}$ \\
\hline \multicolumn{4}{|c|}{ Bloques huecos experimentales } \\
\hline B-0 & 5,93 & 18,12 & 2660 \\
\hline B-33 & 5,00 & 19,71 & 2600 \\
\hline B-39 & 5,24 & 18,77 & 2610 \\
\hline B- 45 & 6,23 & 19,17 & 2630 \\
\hline \multicolumn{4}{|c|}{ Bloques huecos comerciales } \\
\hline $\mathrm{BC} 1$ & 6,30 & 24,33 & 2650 \\
\hline $\mathrm{BC} 2$ & 6,97 & 22,73 & 2680 \\
\hline \multicolumn{4}{|c|}{ Adoquines tipo hueso } \\
\hline Código & $\begin{array}{l}R_{F} 28 d \\
(\mathrm{MPa})\end{array}$ & Absorción, (\%) & $\begin{array}{c}\text { Densidad aparente } \\
\left(\mathrm{kg} / \mathrm{m}^{3}\right)\end{array}$ \\
\hline A- 50 & 4,65 & 19,38 & 2630 \\
\hline
\end{tabular}

B-\#: Bloque-\%FCC; BCl, BC2: Muestras de bloques comerciales; A-\%: adoquín-\%FCC;

$R_{i}$ : Resistencia a compresión; $R_{F}$ : Resistencia a flexotracción.

Fuente: presentación propia de los autores

Los resultados de la tabla 3 muestran que los bloques B 45 superan en resistencia máxima a los bloques B0, mientras que los bloques B33 y B39 alcanzan resistencias del orden de 84 y $88 \%$, respectivamente comparadas a las reportadas por B0. Respecto a los bloques comerciales (BC1 y BC2), la resistencia es comparable a la obtenida en el bloque B45.

Cabe anotar que el cemento utilizado en la mezcla presenta una adición de tipo calcárea, lo cual reduce la disponibilidad de hidróxido de calcio en la mezcla, llevando a que todo el FCC presente pueda actuar como puzolana, por lo cual, parte del FCC permanece como material inerte o relleno en la mezcla. 
Esto implica que a altos porcentajes de adición, el FCC actúa parcialmente por efecto químico (acción puzolánica), y el resto, por efecto físico.

Este último efecto también podrá ser positivo, en la medida en que, acorde con la distribución de tamaño de partícula, complemente los huecos disponibles dejados por los áridos más grandes, creando mayores puntos de contacto y contribuya a densificar aún más el material, lo cual ha sido evidenciado por otros investigadores [36].

Según las normas técnicas colombianas [47], [48], cuyas especificaciones son incluidas en la tabla 4 , los bloques B 45 cumplen con la mínima resistencia a compresión requerida para ser clasificados como 'bloques no estructurales'; a su vez, los adoquines A50 cumplen la resistencia a flexotracción especificada en la norma correspondiente y clasifican como 'adoquines de piso'. Taha et al. [18] produjeron bloques huecos adicionados con FCC al $15 \%$ y reportaron $11,4 \mathrm{MPa}$ a 56 días de curado, valor que, según explican los autores, excede el mínimo de resistencia requerido por bloques de mampostería en los estándares de su país (7,5 $\mathrm{MPa}$ para bloques estructurales y 3,5 $\mathrm{MPa}$ para bloques no estructurales, con dimensiones de $390 \times 190 \times 190 \mathrm{~mm}$ ). Aunque este resultado supera lo reportado en la presente investigación y los productos difieren en sus características físicas, es de anotar que una ventaja del presente estudio es la utilización de elevados porcentajes del FCC en la mezcla, lo cual reduce el consumo de cemento y, por lo tanto, presenta beneficios técnicos, económicos y ambientales.

Tabla 4. Especificaciones técnicas colombianas para los elementos constructivos

\begin{tabular}{|c|c|c|c|c|c|}
\hline \multicolumn{2}{|c|}{ Bloques no estructurales (NTC 4076) } & \multicolumn{3}{c|}{ Adoquines de piso (NTC 2017) } \\
\hline \multicolumn{2}{|c|}{$\begin{array}{c}\mathrm{R}_{\mathrm{C}} \text { mín. } \\
(\mathrm{MPa})\end{array}$} & $\begin{array}{c}\text { Absorción } \\
\text { máx. (\%) }\end{array}$ & \multicolumn{2}{|c|}{$\begin{array}{c}\mathrm{R}_{\mathrm{F}} \text { mín. } \\
(\mathrm{MPa})\end{array}$} & $\begin{array}{c}\text { Absorción } \\
\text { máx. (\%) }\end{array}$ \\
\hline $\begin{array}{c}\text { Promedio } \\
\text { (3 esp.) }\end{array}$ & Espécimen & $\begin{array}{c}\text { Promedio } \\
(3 \text { esp.) }\end{array}$ & $\begin{array}{c}\text { Promedio } \\
(3 \text { esp.) }\end{array}$ & Espécimen & $\begin{array}{c}\text { Promedio } \\
\text { (3 esp.) }\end{array}$ \\
\hline 6 & 5 & 12 & $4,2-5,0$ & $3,8-4,2$ & 7 \\
\hline
\end{tabular}

$R_{i}$ : Resistencia a compresión; $R_{F}$ : Resistencia a flexotracción. Fuente: presentación propia de los autores

Respecto a los valores de absorción de agua, tanto para los bloques como para los adoquines incluidos en la tabla $3(18,12 \%$ y $19,71 \%$, independiente del porcentaje de adición), los elementos constructivos producidos en la presente investigación no cumplen lo especificado en las normas: $12 \%$ máximo (tabla 4); sin embargo, cabe resaltar que los bloques comerciales $\mathrm{BC} 1$ y $\mathrm{BC} 2$ tampoco cumplen esta norma, e incluso superan ampliamente los valores reportados por los elementos aquí desarrollados $(22,73 \%$ y $24,33 \%$, respectivamente). 


\subsection{Caracterización de los productos de bidratación}

La figura 8 presenta el espectro DRX de las mezclas OPC/FCC-1 con porcentajes de sustitución de 0,33 y $50 \%$. En este espectro se aprecian los picos característicos de los productos de hidratación: etringita (E), aluminato cálcico hidratado (CA), portlandita $(\mathrm{CH})$, tobermorita $(\mathrm{T})$, así como los correspondientes a los componentes del cemento sin hidratar, como silicatos bicálcicos (C2) y silicatos tricálcicos (C3), y algunas fases inertes, como cuarzo (Q) y calcita (C), provenientes de la adición caliza presente en el cemento utilizado.

La desaparición completa de los picos $\mathrm{CH}$ para la mezcla con $33 \%$ de FCC-1 indica que un porcentaje superior al $33 \%$ actuaría parcialmente como relleno en la mezcla, lo cual corrobora la reducción en resistencias mecánicas [25]. El aumento de los picos de $\mathrm{E}$ concuerda con la desaparición de los picos de $\mathrm{CA}$; a su vez la disminución los picos C2 y C3 se puede atribuir al efecto de dilución del cemento Portland y al avance en el proceso de hidratación.

Figura 8. Espectro DRX para mezclas $\mathrm{OPC} / \mathrm{FCC}-1(0,33$ y $50 \%)$ a 28 días de curado

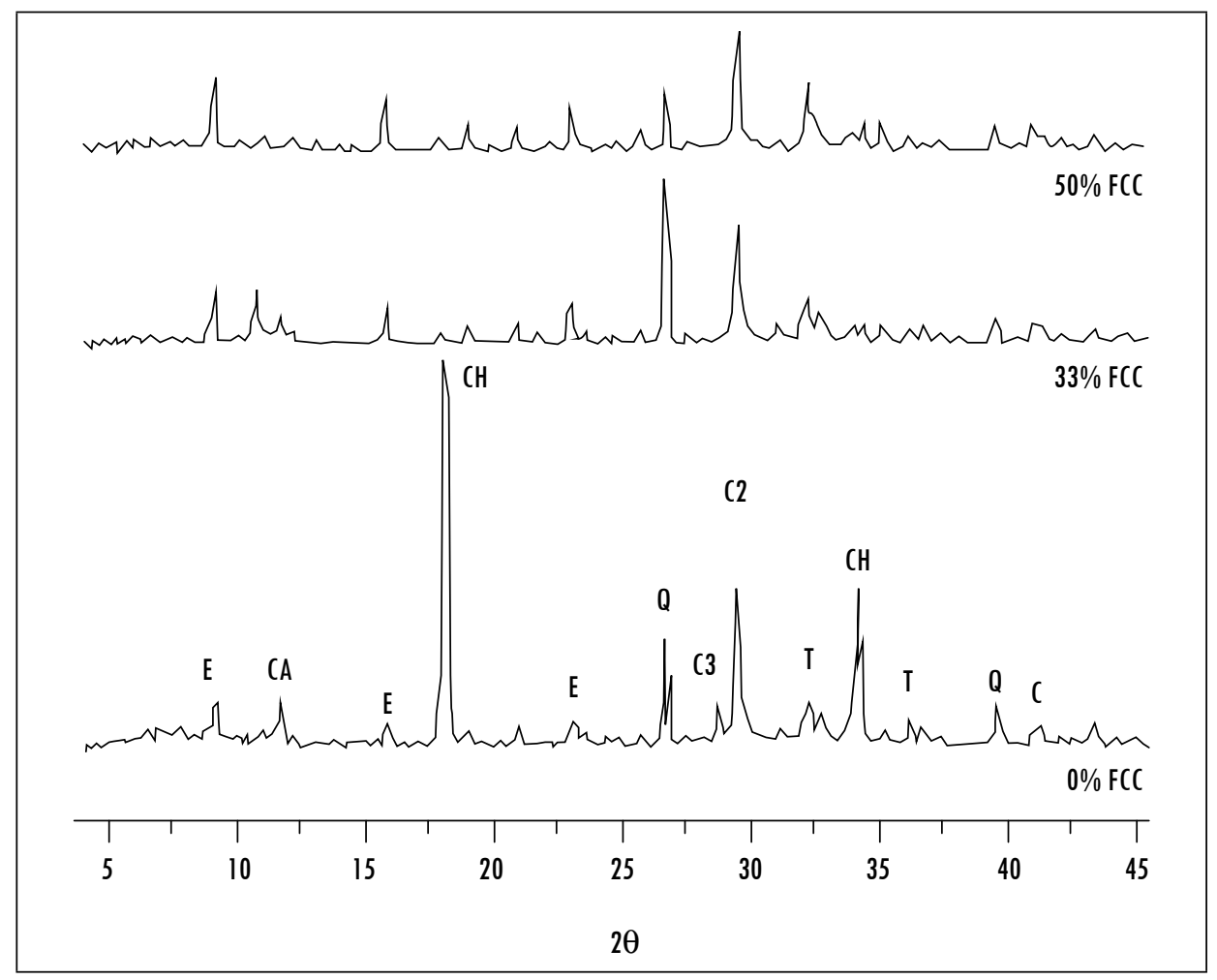

Fuente: presentación propia de los autores

Ing. Univ. Bogotá (Colombia), 19 (1): 135-154, enero-junio de 2015 
La figura 9 muestra la curva DTG para las mismas pastas OPC/FCC-1 evaluadas por DRX; en esta se puede observar la presencia de cuatro picos característicos de este tipo de sistemas. El pico 1 es el pico atribuido a la desintegración de los geles de tipo CSH que se solapa con el pico 2, que es atribuido a la desintegración de la E. El pico 3 corresponde a la desintegración de los aluminatos cálcicos hidratados y los silico-aluminatos cálcicos hidratados. El pico 4 corresponde a la desintegración de la portlandita. Por último, se encuentra el pico 5, que se debe a la decarbonatación de la caliza presente en el tipo de cemento Portland utilizado.

Se observa que a medida que se aumenta el porcentaje de FCC-1 en la mezcla, se aumenta la intensidad de los picos solapados 1 y 2, debido a la mayor formación de geles CSH y de E. La formación de E se ve acompañada de la disminución del pico 3, el correspondiente a los aluminatos cálcicos hidratados. La disminución mostrada por el pico 4 se refleja en el consumo de portlandita en la reacción puzolánica, lo cual concuerda con el resultado observado en los ensayos de DRX [41]. La disminución mostrada por parte del pico 5 se atribuye al efecto de dilución del cemento a medida que aumenta el porcentaje de FCC-1 en la mezcla.

Figura 9. Curvas DTG para sistemas Cemento-FCC-1, 0, 33 y 50 \%

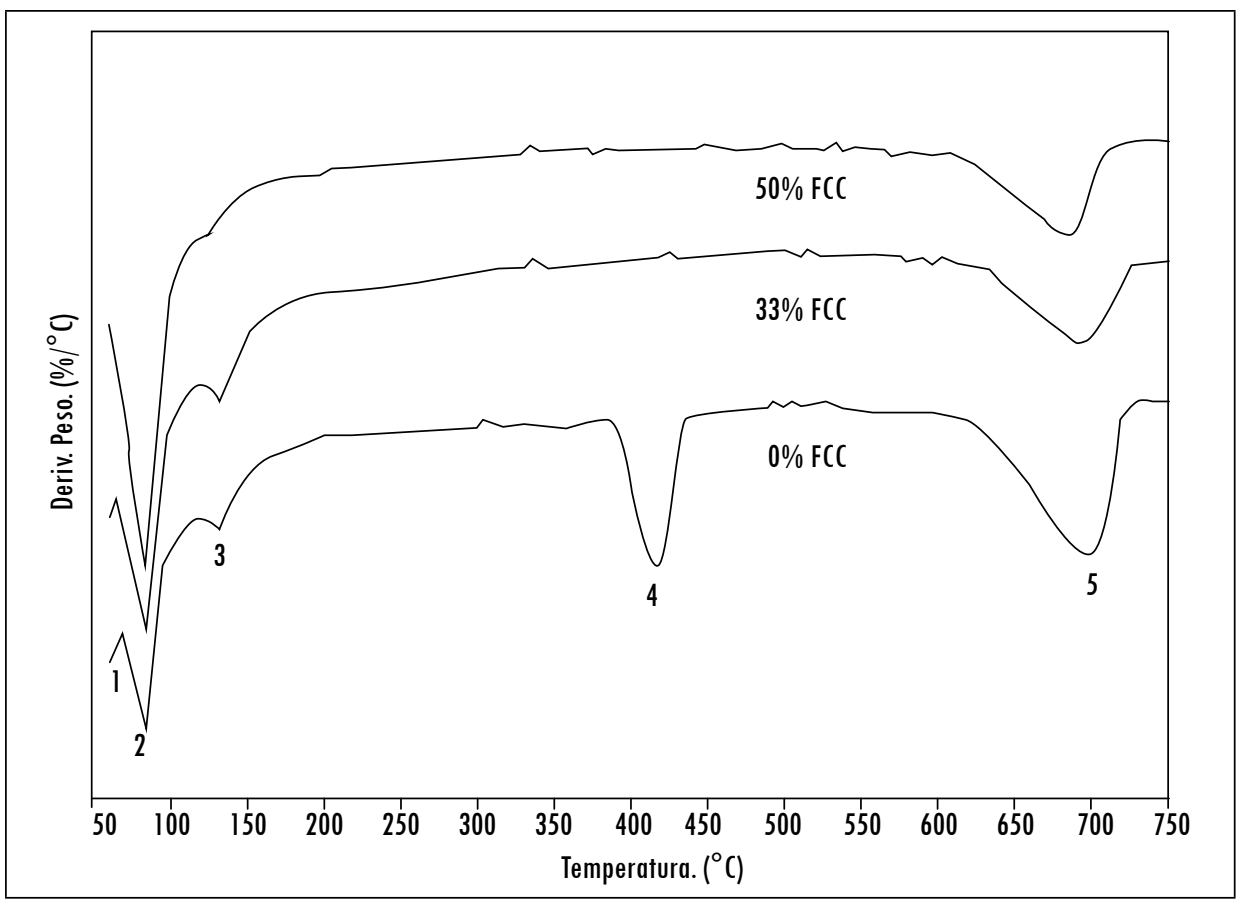

Fuente: presentación propia de los autores 


\section{Conclusiones}

Los resultados de la presente investigación permiten concluir que es viable la utilización de altos porcentajes de FCC como reemplazo del cemento para producir elementos constructivos, como bloques y adoquines, con propiedades comparables a los elementos disponibles comercialmente.

El análisis estadístico de los resultados determinó como proporciones óptimas de mezcla, para alcanzar una resistencia superior a la del OPC utilizado, $67 \%$ OPC y $33 \%$ FCC; esta mezcla reporta un índice resistente de $106 \%$ a 28 días de curado. Los ensayos de TG/DTG y DRX corroboran que en el presente estudio el máximo porcentaje que se debe incorporar de FCC es $33 \%$ para que actué como puzolana; porcentajes por encima de este valor ejercen acción de tipo físico, e igualmente contribuyen a resistencias mecánicas aceptables. Así, un porcentaje de adición de $45 \%$ reporta un $75 \%$ de la resistencia del cemento de referencia a edad de 28 días de curado, lo cual cumple las especificaciones de la norma ASTM C618.

Bloques producidos con OPC/FCC en proporción $55 \% / 45 \%$ y adoquines producidos con OPC/FCC en proporción $50 \% / 50 \%$ cumplen la resistencia mecánica exigida en las normas colombianas para ser usados como bloques no estructurales y adoquines de piso. Respecto a los porcentajes de absorción, aunque superan los especificados en la norma respectiva, resultaron ser inferiores a los disponibles comercialmente. Cabe anotar, que los porcentajes máximos de incorporación de FCC aquí expresados pueden variar en función del tipo de cemento utilizado en la mezcla.

Estos resultados complementan información anterior acerca de que, además de concretos de alto desempeño con bajos porcentajes de adición (menor al $20 \%$ ), es posible incorporar mayores porcentajes de FCC en la producción de algunos tipos de elementos constructivos cuyas resistencias sean moderadas.

\section{Referencias}

[1] E. M. Gartner y D. Macphee, "A physico-chemical basis for novel cementitious binders", Cement Concrete Research, vol. 41, no. 7, pp. 736-749, 2011.

[2] H. G. Van Oss. (2014). U.S. Geological survey, mineral commodity summaries [En línea]. Disponible en: http://minerals.usgs.gov/minerals/pubs/commodity/cement/mcs-2014-cemen.pdf

[3] R. Siddique y J. Klaus, "Influence of metakaolin on the problems of mortar and concrete: A review", Applied Clay Science, vol. 43, no. 3-4, pp. 392-400, 2008.

[4] J. Torres y R. Mejía de Gutiérrez, "Influencia de la composición mineralógica de los caolines sobre el desempeño de morteros adicionados", Dyna, vol. 74, no. 153, pp. 61-67, 2007. 
[5] J. Torres y R. Mejía de Gutiérrez, "Desempeño de morteros adicionados con metacaolín frente a la acción de sulfatos”, Ingeniería Investigación, vol. 28, no. 1, pp. 117-122, 2008.

[6] J. Torres et al., "Procesos de hidratación de pastas OPC adicionadas con caolín tratado térmicamente", Rev. Fac. Ing. Univ. Antioquia, no. 43, pp. 77-85, 2008.

[7] A. Elahi et al., "Mechanical and durability properties of high performance concretes containing supplementary cementitious materials", Construction Building Materials, vol. 24, no. 3, pp. 292-299, 2010.

[8] M. A. Megat et al., "Influence of supplementary cementitious materials on engineering properties of high strength concrete", Construction Building Materials, vol. 25, no. 5, pp. 2639-2648, 2011.

[9] M. Zain et al., "Production of rice husk ash for use in concrete as a supplementary cementitious material", Construction Building Materials, vol. 25, no. 2, pp. 798-805, 2011.

[10] M. Thomas, "The effect of supplementary cementing materials on alkali-silica reaction: A review", Cement Concrete Research, vol. 41, no. 12, pp. 1224-1231, 2011.

[11] B. Lothenbach, K. Scrivener y R. Hooton, "Supplementary cementitious materials", Cement Concrete Research, vol. 41, no. 12, pp. 1244-1256, 2011.

[12] M. Sahmaran, G. Yildirim y T. Erdem, "Self-healing capability of cementitious composites incorporating different supplementary cementitious materials", Cement Concrete Composites, vol. 35, no. 1, pp. 89-101, 2013.

[13] A. Mardani-Aghabaglou, G. Sezer y K. Ramyar, "Comparison of fly ash, silica fume and metakaolin from mechanical properties and durability performance of mortar mixtures view point", Construction Building Materials, vol. 70, pp. 17-25, 2014.

[14] M. Cyr et al., "Effect of cement type on metakaolin efficiency", Cement Concrete Research, vol. 64, pp. 63-72, 2014.

[15] O. Oueslati y J. Duchesn, "Resistance of blended cement pastes subjected to organic acids: Quantification of anhydrous and hydrated phases", Cement Concrete Composites, vol. 45, pp. 89-101, 2014.

[16] S. Antiohos, V. Papadakis y S. Tsimas, "Rice husk ash (RHA) effectiveness in cement and concrete as a function of reactive silica and fineness", Cement Concrete Research, vol. 61-62, pp. 20-27, 2014.

[17]. N. Saca y M. Georgescu, "Behavior of ternary blended cements containing limestone filler and fly ash in magnesium sulfate solution at low temperature", Construction Building Materials, vol. 71, pp. 246-253, 2014.

[18] R. Taha, et al., "Recycling of waste spent catalyst in road construction and mansory blocks", Journal Hazardous Materials, vol. 229-230, pp. 122-127, 2012.

[19] E. Zornosa, J. Paya y P. Garcés, "Chloride-induced corrosion of steel embedded in mortars containing fly ash and spent cracking catalyst", Corrosion Science, vol. 50, no. 6, pp. 1567$1575,2008$. 
[20] J. Amaya, A. Tristancho y C. Sánchez, "Utilizacion de ceniza volante y catalizador de FCC gastado en la recuperación de cromo III de los efluentes liquidos de las curtiembres", Ingeniería Investigación, vol. 25, no. 1, pp. 39-48, 2005.

[21] E. Furimsky, "Review Spent refinery catalysts: environment, safety and utilization", Catalysis Today, vol. 30, no. 4, pp. 223-286, 1996.

[22] N. Su et al., "Reuse of waste catalysts from petrochemical industries for cement substitution", Cement Concrete Research, vol. 30, no. 11, pp. 1773-1783, 2000.

[23] A. Escardino et al., "Utilizing the used catalyst from refinery FCC units as a substitute of kaolin in formulating ceramic frits", Waste management Research, vol. 13, no. 5, pp. 569578, 1995.

[24] E. Sánchez, Aprovechamiento del catalizador usado de FCC de refinerias. Castellón, España: Universitat Jaume I De Castelló, 1994.

[25] J. Trochez, J. Torres y R. Mejáa de Gutierrez, "Estudio de la hidratación de pastas de cemento adicionadas con catalizador de craqueo catalítico usado (FCC) de una refinería colombiana”, Rev. Fac. Ing. Univ. Antioquia, vol. 55, pp. 26-34, 2010.

[26] J. Payá, J. Monzó y M. Borrachero, "Physical, chemical and mechanical properties of fluid catalytic cracking catalyst residue (FC3R) blended cements", Cement Concrete Research, vol. 31, no. 1, pp. 57-61, 2001.

[27] J. Payá et al., "Determination of the pozzolanic activity of fluid catalytic cracking residue. Thermogravimetric analysis studies on FC3R-lime pastes”, Cement Concrete Research, vol. 33, no. 7, pp. 1085-1091, 2003.

[28] J. Payá et al., "Hormigones blancos: nuevos composites con adición de residuo de catalizador de craqueo catalítico”, en Congreso MATCOMP’07, Valladolid, España, 2007.

[29] M. García de Lomas, M. Sánchez de Rojas y M. Frías, "Pozzolanic reaction of a spent fluid catalytic cracking catalyst in FCC-cement mortars", Journal Thermal Analysis Calorimetry, vol. 90, no. 2, pp. 443-447, 2007.

[30] E. Zornoza et al., "Compatibility of fluid catalytic cracking catalyst residue (FC3R) with various types of cement", Advances Cement Research, vol. 19, no. 3, pp. 117-124, 2007.

[31] E. Zornoza et al., "Improvement of the chloride ingress resistance of opc mortars by using spent cracking catalyst", Cement Concrete Research, vol. 39, no. 2, pp. 126-139, 2009.

[32] B. Pacewska, I. Wilinska y J. Kubissa, "Use of spent catalyst from catalytic cracking in fluidized bed as a new concrete additive", Thermochimica Acta, vol. 322, no. 2, pp. 175-181, 1998.

[33] B. Pacewska, I. Wilinska y M. Bukowska, "Hydration of cement slurry in the presence of spent cracking catalyst", Journal Thermal Analysis Calorimetry, vol. 60, no. 1, pp. 71-78, 2000. 
[34] B. Pacewska, I. Wilinska y M. Bukowska, "Influence of some aggressive media on corrosion resistance of mortars with spent cracking catalyst", Journal Thermal Analysis Calorimetry, vol. 60, no. 1, pp. 257-264, 2000.

[35] B. Pacewska et al., "Modification of the properties of concrete by new pozzolan A waste catalyst from the catalytic process in a fluidized bed", Cement Concrete Research, vol. 32, no. 1, pp. 145-152, 2002.

[36] K. Al-Jabri et al., "Potential use of FCC spent Catalyst as partial replacement of cement or sand in cement mortars", Construction Building Materials, vol. 39, pp. 77-81, 2013.

[37] R. Neves et al., "Durability performance of concrete incorporating spent fluid cracking catalyst”, Cement Concrete Composites, vol. 55, pp. 308-314, 2015.

[38] M. Morsy et al., "Behaviour of blended cement mortars containing nano-metakaolin at elevated temperatures”, Construction Building Material, vol. 35, pp. 900-905, 2012.

[39] H. Wu et al., "The effect of waste oil-cracking catalyst on the compressive strength of cement pastes and mortars", Cement Concrete Research, vol. 33, no. 2, pp. 245-253, 2003.

[40] Y. Tseng, C. Huang y K. Hsu, "The pozzolanic activity of a calcined waste FCC catalyst and its effect on the compressive strength of cementitious materials", Cement Concrete Research, vol. 35, no. 4, pp. 782-787, 2005.

[41] J. Torres, A. Baquero y A. Silva, "Evaluación de la actividad puzolanica de un residuo de la industria del petroleo", Dyna, vol. 76, no 158, pp. 49-53, 2009.

[42] J. Torres, J. Trochez y R. Mejía de Gutiérrez, "Reutilización de un residuo de la industria petroquímica como adición al cemento portland", Ingeniería Ciencia, vol. 8, no. 15, pp. 141-156, 2012.

[43] Colombia, Icontec, Norma Técnica Colombiana (NTC) 111. Especificaciones para la mesa de flujo usada en ensayos de cemento bidráulico. Bogotá: Icontec, 2013.

[44] Colombia, Icontec, Norma Técnica Colombiana (NTC) 4024. Prefabricados de concreto. Muestreo $y$ ensayo de prefabricados de concreto no reforzados, vibrocompactados. Bogotá: Icontec, 2001.

[45] S. Antiohos, E. Chouliara y S. Timas, "Re-use of spent catalyst from oil-cracking refineries as supplementary cementing material”, China Particuology, vol.4. no. 2, pp. 73-76, 2006.

[46] B. Pacewska, et al., "Early hydration of calcium aluminate cement blended with spent FCC catalyst at two temperatures", Procedia Engineering, vol. 57, pp. 844-850, 2013.

[47] Colombia, Icontec, Norma Técnica Colombiana (NTC) 2017. Adoquines de concreto para pavimentos. Bogotá: Icontec, 2004.

[48] Colombia, Icontec, Norma Técnica Colombiana (NTC) 4076. Unidades (bloques y ladrillos) de concreto, para mampostería no estructural interior y chapa de concreto. Bogotá: Icontec, 1997. 